\title{
Positive Affect, Negative Affect, and the Moderating Effect of Writing on Siga Antibody Levels
}

David Michael Njus

Loyola University Chicago

Follow this and additional works at: https://ecommons.luc.edu/luc_theses

Part of the Psychology Commons

\section{Recommended Citation}

Njus, David Michael, "Positive Affect, Negative Affect, and the Moderating Effect of Writing on Siga Antibody Levels" (1992). Master's Theses. 3917.

https://ecommons.luc.edu/luc_theses/3917

This Thesis is brought to you for free and open access by the Theses and Dissertations at Loyola eCommons. It has been accepted for inclusion in Master's Theses by an authorized administrator of Loyola eCommons. For more information, please contact ecommons@luc.edu. (c) (i) $(9)$

This work is licensed under a Creative Commons Attribution-Noncommercial-No Derivative Works 3.0 License. Copyright @ 1992 David Michael Njus 

POSITIVE AFFECT, NEGATIVE AFFECT, AND THE MODERATING EFFECT OF
WRITING ON SIGA ANTIBODY LEVELS

David Michael Njus

A Thesis Submitted to the Faculty of the Graduate School of Loyola University of Chicago in Partial Fulfillment of the Requirements for the Degree of Master of Arts

May 
Copyright, 1992, David M. Njus

All rights reserved.

i i 


\section{Acknowledgements}

I would like to thank the many individuals who have offered their assistance and support toward the completion of this work. I especially appreciate the time, help, and advice provided by Dr. Werner Nitschke and Dr. Fred Bryant.

Special thanks to Dr. Bernard Dugoni, whose statistical and computer assistance and hours of patient support were invaluable. 
To my alma mater and the mission it professes SOLI DEO GLORIA 
COPYRIGHT PAGE

ACKNOWLEDGEMENTS

TABLE OF CONTENTS

LIST OF TABLES AND FIGURES

I. INTRODUCTION • • . . . . . . . . . . . . . . . 1

Overview of the Immune System . . . . . . . 2

Types of Immunity . . . . . . . . . . . 2

Humoral Immunity and the Immuno-

$$
\text { globulins . . . . . . . . . . . } 3
$$

Secretory Immunoglobulin $A$ as a

Measure of Immunocompetence

Health, the Immune System, and Environmental

Factors . . . . . . . . . . . . . . .

Negative Affect and Stressors . . . .

Positive Affect. . . . . . . . . . . .

Moderation of Affect and Immuno-

competence

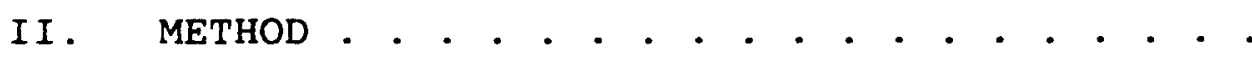

Study I

Study I I

Subjects

Apparatus and Materials . . . . . . . 14

Procedure . . . . . . . . . . . . 15

Assessing sIgA Concentrations . . . . . 16

Research Hypotheses . . . . . . . . 17

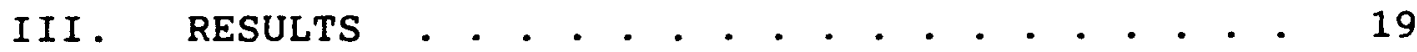

Manipulation Check . . . . . . . . . . . 19

Essay Characteristics . . . . . . . . . . 19

Immunological Data . . . . . . . . . . 23

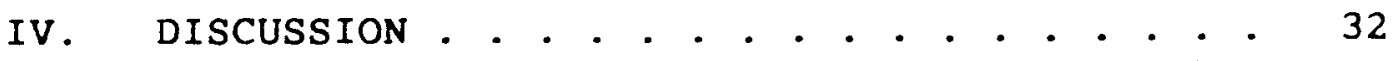

Processes of Moderation . . . . . . . . 36 
The Process Underlying Negative Affect

Writing . . . . . . . . . . . . 37

Support for the Theory . . . . . . . . 39

The Process Underlying Humor Writing . 40

Neuroimmunoendocrinology and the Moderating

Processes . . . . . . . . . . . 42

Questions and Answers Regarding the Current

Study .. . . . . . . . . . . . 46

Directions for Future Research . . . . . . 49

REFERENCES . . . . . . . . . . . . . . . . . 52

VITA . . . . . . . . . . . . . . . 57 


\section{LIST OF TABLES AND FIGURES}

Table

1. Pretest and Posttest Affect Ratings . . . 20

2. Mean sIgA Precipitin Ring Diameters for Positive and Negative Affect Groups . . . . 26

3. Source Table for Time $x$ Gender $x$ Movie $\mathbf{x}$ Writing Condition ANCOVA . . . . . . . . . 28

4. Male and Female sIgA Precipitin Ring Diameters for the Positive Affect Group . . 30

Figure

1. Mean Number of Words Written in Video and Writing Groups . . . . . . . . . . . . 22

2. Mean Number of Sentences Written in Essays Written About the Videos......... . 24

3. Conceptualization of the interaction among the immune, limbic, neuroendocrine, and central nervous systems . . . . . . . . . 


\section{Introduction}

The link between psychological and physical well-being is an intuitive one, and one that is rapidly gaining scientific support. Many areas of psychology, however, including social psychology with its emphasis on information processing models of social cognition, have been slow to recognize the ties that bind physiological and psychological well-being. Indeed, Pennebaker (1989) states that the cognitive revolution in social psychology is "running low on ammunition" partly because cognitive social psychologists ignore the fact that information processing occurs not simply in the "mind," but within a complex biochemical organ, the brain. Any understanding of the mind must acknowledge the indivisibility of the mind and body, must go beyond mere psychological models to biopsychosocial models, where an integration of the whole person with their environment can be explored.

One area of particular interest from a biopsychosocial perspective concerns the human immune system and how it responds to psychological and environmental factors. The focus of the following research concerns how everyday stimuli, both positive and negative, can affect the functioning of the immune system. Additionally, I explore the psychological processes which may moderate the relation- 
ship between the immune system and these environmental stimuli.

Overview of the Immune System

Before proceeding further with a review of relevant literature, it is necessary to give a brief overview of the human immune system, particularly those aspects of the immune system relevant to this study.

\section{Types of Immunity}

In his discussion of immunity, Guyton (1991) outlines two basic kinds of immunity--innate and acquired. Innate immunity refers to general processes, such as resistance of the skin to invasion by foreign organisms, and phagocytosis (surrounding, encapsulating, and digesting) of bacteria and other invaders by white blood cells. Acquired immunity refers to the body's efforts to attack a specific organism or toxin, such as the chicken pox virus.

Acquired immunity is further divided into humoral and cell-mediated immunity. T lymphocytes, the major elements of cell-mediated immunity, are antigen-specific. (Antigens are the unique protein coatings present on invading organisms.) Antigen-specificity refers to the ability of a particular lymphocyte to react to only one type of antigen, the chicken pox virus for example, and no other. When $T$ lymphocytes come into contact with foreign organisms, they differentiate into different types of $T$ cells which can kill the organisms outright, regulate cell-mediated immunity, and assist in the phagocytic process. 
Humoral Immunity and the Immunoglobulins

The primary agents of humoral immunity are the $B$ Iymphocytes, which, like T lymphocytes, are products of the lymph system. Also like T lymphocytes, B lymphocytes are antigen-specific. Unlike $T$ lymphocytes, however, $B$ lymphocytes do not directly fight invading organisms.

B lymphocytes are stored in the lymphoid tissue until they come into contact with their particular antigen, at which time they enlarge and become lymphoblasts. Lymphoblasts further differentiate into plasmablasts, which in turn begin creating plasma cells at the rate of about 125 cells per day. It is the plasmablasts that then begin creating antibodies at the rate of about 2000 every second. These antibodies, called immunoglobulins, are the agents that work directly to combat the organism that has invaded the body. There are 5 classes of immunoglobulins: IgA, IgD, IgE, IgG, and IgM, each of which protects the body either by attacking invading agents directly or by activating the complement system, which in turn destroys or inactivates the agent.

Secretory Immunoglobulin A as a Measure of Immunocompetence

IgA is the immunoglobulin normally examined in psychological studies where antibody levels or performance are used to reflect immune system functioning. Serum IgA, IgA present in circulating blood, exists only in very small amounts compared to other immunoglobulins. Secretory IgA (sIgA), on the other hand, is the dominant antibody present 
in saliva, tears, gastrointestinal and respiratory secretions, and the body's mucous membranes.

The advantages of using SIgA as a measure of immunocompetence are threefold. First, because sIgA is present in saliva, it is easier, less painful, and less stressful to extract than are the immunoglobulins found in the blood. Second, since sIgA is considered to be the body's first line of defense against invading organisms, it is believed to be an important factor in determining the health and well-being of the individual. Lastly, sIgA is considered to be most effective in combatting upper respiratory illness, a type of illness common among the college populations who comprise the subject pools in much psychological research.

Since the immune system is complex and consists of many components capable of combatting antigenic substances, it is very difficult to directly link sIgA levels to the occurrence of illness. Nonetheless, several studies have shown that lower levels of SIgA are associated with more frequent illness. Rossen, et al. (1970) infected 15 subjects with one of two types of influenza virus. Flu symptoms developed in those subjects with lower baseline levels of SIgA, and influenza-neutralizing antibody was discovered earlier and reached highest levels sooner in subjects with high preinoculation sIgA levels.

In a second study relating sIgA levels with illness, Yodfat and Silvian (1977) used 48 children living in an Israeli kibbutz as subjects. They found that while baseline 
levels of SIgA (when children were healthy) were not associated with the number of acute respiratory infections the children had in a year, sIgA levels during respiratory infections were. Children possessing higher sIgA levels when they did have infections had fewer infections throughout the year.

In a study with 27 male college students as subjects, McClelland, Floor, Davidson, and Saron (1980) found that sIgA concentrations were uncorrelated with the number of illnesses subjects had in the past 6-10 months. However, subjects with sIgA concentrations below the median did have significantly more illnesses in the past 6-10 months than subjects with sIgA levels above the median.

Lastly, in a study of 133 male prison inmates, McClelland, Alexander, and Marks (1982) examined stress, immune function, and need for power. McClelland and his associates found that subjects with higher sIgA levels reported fewer severe upper respiratory illness symptoms than did subjects with lower sIgA levels. Though the results of these studies do not reveal a definite causal link between sIgA levels and an individual's somatic health, they nevertheless lend support to the proposition that higher sIgA levels result in fewer incidences of at least some types of illnesses.

Health, the Immune System, and Environmental Factors Negative Affect and Stressors 
In his work with stress, Richard Lazarus (1984) has noted the relationship between daily hassles and health. Daily hassles are daily occurrences that the individual appraises as harmful or stressful. Although Lazarus found hassles to be a better predictor of somatic health than life events, he was unable to draw a clear causal link between hassles and health, and was unable to describe the nature of the possible relationship between the two, i.e. whether it is additive or non-linear, whether certain hassles are more devastating than others to physical health, etc.

Other studies have linked stressors to health deficits. McClelland et al. (1982), in addition to linking sIgA to illness as mentioned above, found that high stress subjects had lower concentrations of SIgA and reported greater severity of upper respiratory illnesses and greater severity of all illnesses. Using a one-group pretest-posttest design, Kiecolt-Glaser et at. (1984) found that natural killer cell activity (activity from an immune system antitumor and antiviral lymphoid cell) declined significantly in medical students during a period one month before final exams until the day finals began. Levels of plasma antibody Immunoglobulin $A$, however, increased significantly, while plasma IgG and IgM, and SIgA levels did not change significantly. It is difficult to draw conclusions from these seemingly contradictory results, especially inasmuch as there is such a long period of time between pretest and posttest, and because the methodologically weak experimental 
design lacked a control group to which comparisons might have been made.

In another one-group study, this one with one pretest and two posttest measures, Schleifer, Keller, Camerino, Thornton, and Stein (1983) tested lymphocyte reactions in subjects whose spouses died during the course of the experiment. They found that lymphocyte stimulation and function were lower during the bereavement period when compared to the pre-bereavement period. Again, however, the results are not as conclusive as they might be because of the absence of a control group.

Jemmott and Magliore (1988) assessed sIgA concentrations of 15 college students during a high stress period (fall semester final exams) and at less stressful periods five days before the exams and at the beginning of the spring semester. Results showed that, as had been predicted, sIgA levels were lower during the exam period than at the pre- and post-exam periods considered together. Taken together, these studies indicate that stress and negative affect are related not only to immediate immunodeficiency, but to health problems over a longer period of time.

Positive Affect

Research has also been conducted on the events and conditions that can impact positively on the immune system. For example, Stone, Cox, Valdimarsdottir, Jandorf, and Neale (1987) found a relationship between daily mood and potential 
consequences for health. In a study of dental students, saliva was collected three times a week for an eight-week period and was checked for levels of sIgA. Stone and his associates found that sIgA response to an antigen was lower on days with high negative mood compared to days with lower negative mood, and that sIgA response was higher on days with high positive mood compared to days with low positive mood. This evidence suggests that an individual's day-today mood can influence immunocompetence and potentially the health of the individual.

Martin and Dobbin (1988) examined the stress-moderating effect of humor on sIgA levels. They used four scales to tap different aspects of sense of humor for 40 subjects at time 1 , and used measures of daily hassles (stress) and sIgA at both time 1 and time 2 , about 6 weeks later. They found a significant correlation between hassles at time 1 and sIgA concentrations at time 2. Using a series of hierarchical multiple regression analyses, Martin and Dobbin found that the magnitude of the relationship between hassles at time 1 and sIgA at time 2 was enhanced by three of the four humor scales. Further examination of these data showed that those scoring high on these 3 scales did not exhibit appreciable sIgA changes as hassles increased, whereas those with low scores on the 3 humor scales demonstrated a marked decline in SIgA concentrations as hassles increased. In other words, daily hassles had little effect on sIgA concentrations for subjects with high scores on three of the four 
humor scales, but for those with low humor scores on those three scales, daily hassles were associated with impaired immunocompetence.

In another investigation of the relationship between emotions and immunological response, Dillon, Minchoff, and Baker (1985-1986) examined the effect of watching a humorous videotape on sIgA levels. Though their sample size was small ( $N=9)$, Dillon et al. found that sIgA concentrations were significantly higher following the humorous video than they were before the video, while watching a control videotape had no such effect on sIgA levels. This corroborates Stone et al.'s (1987) finding that positive mood can enhance immune system functioning. It is important to note, however, that the significant sIgA increase that Dillon and her colleagues found as a result of watching the humorous video declined within minutes of viewing.

McClelland and Kirshnit (1988) also looked at the effects of viewing certain movies on the human immune system. McClelland and Kirshnit had subjects watch movies designed to arouse power motivation (a World War II propaganda $\mathrm{film}$ ) and affiliation motivation (a film on Mother Teresa). Contrary to their predictions, the power motivation film did not lower sIgA concentrations relative to the pretest measure; but, as predicted, the affiliation motivation film did raise sIgA levels significantly. As with Dillon et al.'s 1985-1986 study, however, these 
immunoenhancing effects dissipated within an hour of viewing the film.

Moderation of Affect and Immunocompetence

The research cited above details the effect that various emotional and stressful events can have on the immune system. Recall, however, that the immunoenhancing results of Dillon et al. (1985-1986) and McClelland and Kirshnit (1988) dissipated quickly, and that the deleterious effect on the immune system seems to linger in those who experience stress or negative affect. Assuming for the moment a causal relationship, that is

affect -----> immunological response, is it possible for something to act as a moderator between affect and immunological response? Specifically, is there something that a person can do to prolong the beneficial consequences of positive affect and to reduce or cut short the adverse consequences of negative affect?

Several studies show that there may indeed be a means of moderating affective impact on immunocompetence. With respect to positive affect, McClelland and Kirshnit (1988), after watching Mother-Teresa-movie-enhanced SIgA levels quickly return to baseline, discovered a means of extending the immunoenhancement beyond a few minutes. McClelland and Kirshnit instructed another group to watch the film and then write about it immediately afterwards, specifically to write about what it is like to be cared for by someone like Mother Teresa and about what it is like to love someone in return. 
Unlike the previous non-writing group, this group continued to show significantly higher sIgA levels one hour after viewing the film.

other research demonstrates that the detrimental impact of negative affect on the immune system can be attenuated. Pennebaker and $0^{\prime H e e r o n ~(1984) ~ e x a m i n e d ~ t h e ~ h e a l t h ~ a n d ~}$ coping strategies of 19 spouses of suicide and accidentaldeath victims. Their results indicated that rumination, i.e. subjects thinking about their spouse's death, was significantly and positively correlated with illness rates. The more subjects discussed or confided in others about the death, however, the less likely they were to ruminate about the death, and the less likely they were to become ill.

Pennebaker and Beall (1986) took this research into the laboratory. Forty-six undergraduates wrote for four days about either trivial or personally traumatic experiences. Subjects in the trauma condition wrote about 1) only the facts of what had happened to them, 2) only how they felt about what had happened, or 3) both what had happened and how they felt about it. Results revealed that those who wrote about their emotions and about both the facts and their emotions experienced higher blood pressure and negative affect than the fact-only and trivial-event group; but the emotion and fact-emotion groups also had fewer health center visits and a greater decline in the number of illnesses in the subsequent six months than did the other two groups. 
In a follow-up to this last study, Pennebaker, KiecoltGlaser, and Glaser (1988) investigated the impact of writing about personally traumatic experiences on the immune system and on health. Blood samples were drawn from student subjects immediately before, immediately after, and six weeks after four days of writing about either personally traumatic or mundane topics. These blood samples were tested for lymphocyte response to two different mitogens. Subjects who wrote about traumatic situations showed significantly higher lymphocyte response for one of the mitogens, and reported significantly fewer health center visits than did control subjects. Although subjects who wrote about personally traumatic experiences were significantly less happy than the control subjects immediately following the experiment, they reported being significantly happier than did control subjects at a three month followup. These findings suggest that writing about personally negative or traumatic events can be immunoenhancing. The research presented here continues the study of the affectimmunocompetence link, and explores in greater detail the role that writing may play in moderating this relationship. 


\section{Method}

\section{Study I}

A small pilot study was conducted to examine the effects on the immune system of writing about a negative incident presented in written form. Thirteen subjects read a newspaper article about a young boy who died needlessly from a treatable illness after months of neglect. Seven subjects (experimental group) then wrote about the article, and about how it made them feel, while six subjects (control group) wrote about a mundane topic, their shoes (cf. Pennebaker, et al., 1988). Levels of SIgA from saliva samples taken before reading the article, immediately after writing, 1 hour after writing, and 23 hours after writing were examined using an enzyme-linked immunosorbent assay (ELISA) (Tijssen, 1985). It was hypothesized that levels of sIgA would drop for those subjects unable to write about the article, while sIgA levels would remain constant for subjects asked to write about the article.

Contrary to predictions, control group sIgA levels did not decline significantly at the one hour or twenty three hour test periods, and experimental group sIgA levels did not remain the same but instead increased significantly for these periods. These results are, however, consistent with the basic rationale behind the predictions; those subjects 
who wrote about the article experienced a better immune system response relative to their pre-trial sIgA levels than did those subjects who wrote about the mundane topic.

\section{Study II}

Study II further investigates the moderating effect of writing about a stimulus or event on SIgA levels. The stimulus media was a video tape rather than a newspaper article, and positive affect as well as negative was examined. After watching either an emotionaliy positive or emotionally negative video tape, subjects wrote about either the video or about a mundane topic. Subjects' affect intensity was assessed to determine if intensity was related to sIgA levels.

Subjects

Subjects were 52 undergraduates from a small, liberal arts college who participated for extra credit in an introductory psychology course.

Apparatus and Materials

Subjects viewed one of two videos, each approximately 35 minutes long. The negative affect video was a cutting from an episode of the 48 Hours television program. The episode, "Who Killed Allen Griffen?" was about the same young boy who was the subject of the newspaper article read as a negative affect stimulus in study 1 . The positive affect video, Bill Cosby Live, was a cutting from a performance by comedian Bill Cosby. Subjects' affect intensity was measured by the Affect Intensity Measure (AIM) (Larsen \& 
Diener, 1987). To measure SIgA, saliva was collected in plastic cups and transferred from there into test tubes, which were then covered with parafilm and frozen. After thawing, micrometers were used to place saliva in wells on Low Level IgA Radial Immune Diffusion Endoplates. Procedure

The experiment was run in two simultaneous but separate sessions, with 26 subjects in each session. Each session followed the same order. Subjects were given a short introduction and asked to fill out an informed consent form. Subjects then completed a four-item questionnaire assessing their present feelings of happiness, depression, anger, and distress. Subjects next provided a saliva sample. Each subject expectorated into a plastic cup for five minutes; cups were collected, saliva was transferred to test tubes, and frozen.

Subjects then watched one of the two videos, depending on the particular session to which they had been randomly assigned. Immediately following the video presentation, each subject again completed the four-item questionnaire concerning their feelings. These questions were a manipulation check to determine if subjects found the video they watched to be uplifting or depressing.

All subjects then wrote an essay. According to random assignment, half of the subjects were instructed to write about the video, half about a mundane topic--their shoes 
(Pennebaker et al., 1988). The instructions for the video essay were as follows:

For the next 20 minutes you are to write about the video you have just seen. Write about what happened in the video and how it made you feel. It is important that you write for the full twenty minutes and that you write what you truly think and feel. Whatever you write will be kept strictly confidential.

The instructions for the essay on shoes were as follows:

For the next 20 minutes you are to write about the shoes you are wearing right now. Write about what they look like, where you got them, or whatever you wish, but it is important that you write for the full twenty minutes and that you write only about your shoes. Whatever you write will be kept strictly confidential.

Immediately after finishing the essay, another saliva sample was collected, and subjects were dismissed with instructions to return in one hour. Subjects were also instructed not to speak with anyone during the break about the video they had seen or about the essay they had written. When the subjects returned, another saliva sample was collected. Following this, subjects were dismissed again, with instructions to return in 23 hours (approximately 24 hours after the second saliva sample collection). Subjects were again instructed to speak to no one about the experiment during that 23 hour period. Upon their return the following day, a final saliva sample was collected. Subjects then filled out the AIM (Larsen \& Diener, 1987), were debriefed and then dismissed. Assessing sIgA Concentrations

Saliva was tested for concentrations of sIgA using the single radial immuno-diffusion method (RID) (Martin, 
Guthrie, \& Pitts, 1991; McClelland \& Kirshnit, 1988).

Thawed saliva was centrifuged and 10 microliters of it were placed in wells on low-level IgA endoplates. Each well was pre-coated with monospecific goat anti-human IgA (goat IgA that will react only to human IgA). Each endoplate contained 16 wells; one sample from each of 16 experimental cells ( 2 [video] x 2 [writing condition] $x 4$ [time period]) was placed on each endoplate to balance across conditions in the unlikely event differences between plates existed (if the manufacturers had been erroneously prepared one plate, this error would be balanced across conditions). As the saliva diffused in the well over a 24 hour period, the goat anti-human IgA and the human SIgA formed a precipitin ring, the diameter of which is proportional to the concentration of SIgA in the saliva placed in the well (McClelland \& Kirshnit, 1988). Two researchers measured precipitin ring diameters with a small caliper which electronically displayed measurements in a digital format.

\section{Research Hypotheses}

Hypothesis I, based on McClelland and Kirshnit's (1988) use of writing to sustain elevated SIgA concentrations, is that those who write about the humorous videotape will have higher sIgA concentrations across time than their counterparts who watch the same video but write about the mundane topic. Hypothesis II is that those who write about the depressing video will have higher sIgA levels than their counterparts who watch that video but write about the 
mundane topic. This follows from Pennebaker et al.'s (1988) finding that writing about negative events will attenuate the negative impact those events have upon the immune system. Hypothesis III is that across time, sIgA concentrations for the four groups ( 2 movies $x 2$ writing conditions) will converge. This is based on the assumption that neither stimulus video is that powerful and will have an effect for only a short length of time. In other words, sIgA concentrations of those who write about the humorous video will gradually fall to sIgA concentrations of those who did not write about it, and sIgA concentrations of those who did not write about the negative video will gradually rise to meet the concentrations of those who did write about it.

Hypothesis IV concerns affect intensity, as measured by the AIM. Affect intensity refers to a stable individual difference in how individuals experience emotions, irrespective of the valence of the emotions (Larsen \& Diener, 1987). It is natural to assume that if affect is related to shifts in SIgA concentrations, then the greater the intensity of the affect for a given individual, the more that affect should influence her or his sIgA concentrations. Hypothesis IV is thus that affect intensity scores will be significantly and positively correlated with sIgA concentrations in the Cosby video condition, and significantly and negatively correlated with sIgA concentrations in the Allen Griffen video condition. 


\section{Results}

Before any statistics were performed, each of the essays was read for content. Data from two subjects were discarded, one because the subject did not follow instructions concerning the essay, and another because the subject did not complete the experiment. This left a total of 50 subjects.

\section{Manipulation Check}

Examination of the manipulation check means shown in Table 1 reveals that, as expected, subjects who watched the Allen Griffen (negative) video were less happy, sadder, angrier, and more distressed after watching the video than they were before watching it. Subjects who watched the Bill Cosby video, on the other hand, were happier, less sad, less angry, and less distressed after watching the video than they were before. All eight repeated measures ANOVAs performed on these data were significant, with $p$ values ranging from $\mathrm{p}<.04$ to $\mathrm{p}<.0001$.

\section{Essay Characteristics}

A $2 \times 2$ ANOVA was performed on the number of words written in essays, with video and writing condition as between subjects factors. Significance was not attained for video, $F(1,46)=.46$, n.s., or for writing condition, $F(1,46)=$ .10 , n.s. There was, however, a significant movie $x$ writing 
Table 1 .

Mean affect ratings on a 1 to 7 scale for subjects before and after watching either the Allen Griffen (negative affect) or the Bill Cosby (positive affect) video.

GRIFFEN VIDEO

$$
(n=25)
$$

COSBY VIDEO

$(n=25)$

\begin{tabular}{ccccc} 
Affect & Pretest & Posttest & Pretest & Posttest \\
\hline Happy & 4.76 & 3.24 & 4.32 & 5.28 \\
Sad & 1.80 & 4.36 & 1.96 & 1.48 \\
Angry & 1.84 & 3.92 & 1.68 & 1.28 \\
Distressed & 3.52 & 4.60 & 3.20 & 2.44 \\
\hline
\end{tabular}

Note. Higher scores reflect a stronger endorsement of the particular affect. 
condition interaction, $F(1,46)=3.50, p=.0006$. As Figure 1 shows, those subjects watching the Allen Griffen video and writing about it wrote more words than their counterparts who wrote about their shoes. In the humorous video condition, however, this pattern is reversed; those writing about their shoes wrote more than those who wrote about the video.

In order to determine if subjects in the different video conditions who wrote about the video they had seen wrote about different types of things, each sentence in the video essays was content analyzed on two dimensions. ${ }^{1}$ The first was topic of the sentence--about the video or about the person writing the essay (self); the second dimension was type of sentence--descriptive-objective (sentence was factual in nature), descriptive-subjective (sentence offered an opinion or evaluation), or problem-focused (sentence offered some sort of solution or response to a problem).

A 2 (video) $\times 2$ (sentence topic) $\times 3$ (type of sentence) repeated measures ANCOVA was performed on the number of codeable sentences that were in each category (approximately $9 \%$ of the sentences were not codeable using these categories). Total number of codeable sentences in each essay was

${ }^{1}$ For practical reasons, the experimenter was the only coder of the essay data. This raises certain methodological concerns, especially that of bias. Since there were no a priori hypotheses made about these data, however, and since analyses of the essay data were not central to the study, it was deemed that these exploratory analyses should be conducted and reported anyway, with the understanding that they should be interpreted with caution. 


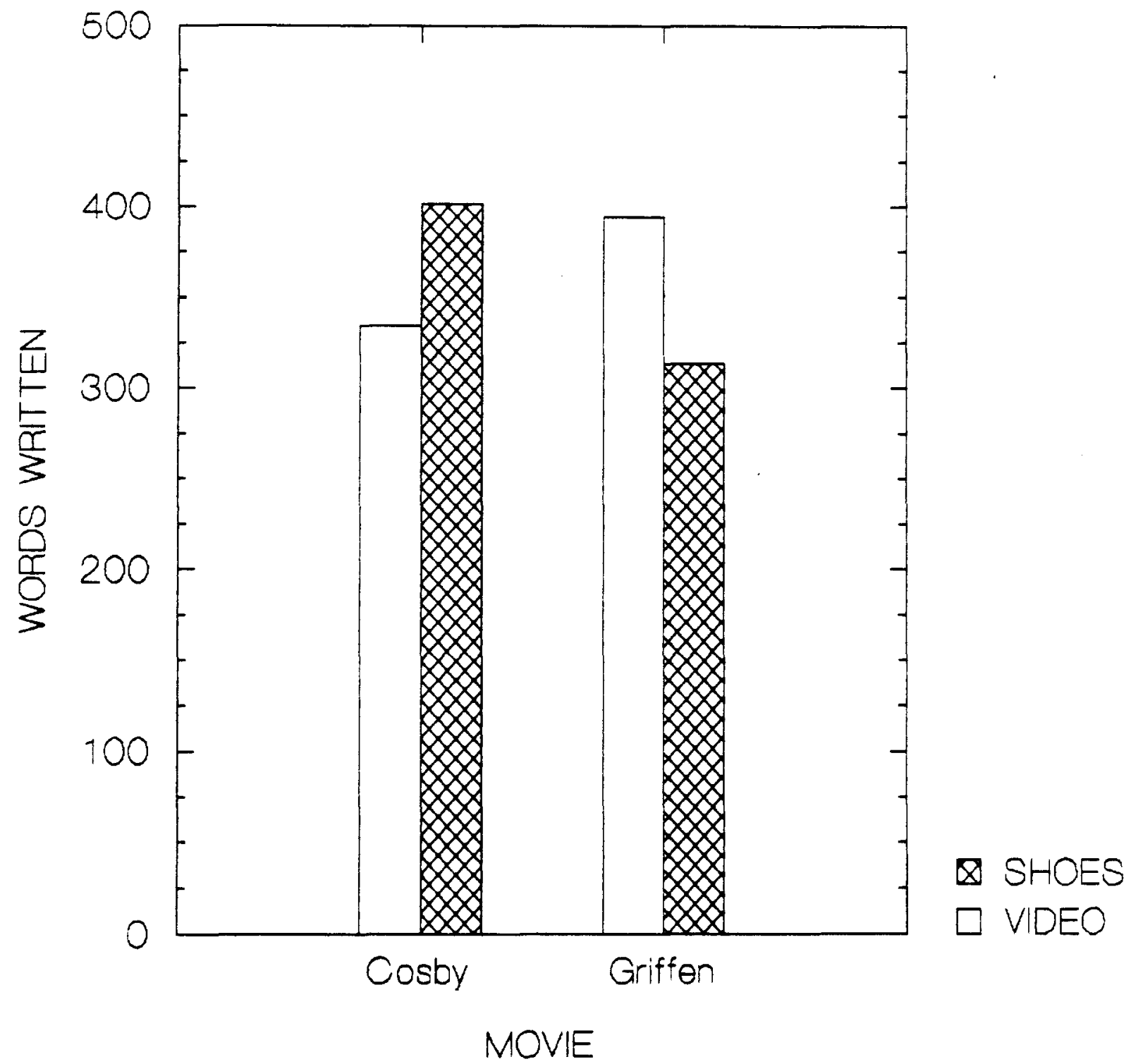

Figure 1.

Mean number of words written by subjects who watched either the Cosby or Allen Griffen video and wrote essays about either the video or their shoes 
the covariate. The results of the ANCOVA indicate that

their were no main effects for movie, $F(1,21)=0.0$, n.s., for topic of sentence, $F(1,21)=.543, \mathrm{n} . \mathrm{s}$. , or for type of sentence, $F(2,21)=1.205$, n.s. In fact the only significant result was a video (Allen Griffen/Cosby) $\mathbf{x}$ type of sentence (descriptive-objective/descriptive-subjective/problemfocused) interaction, $F(2,21)=3.756, p=.032$. As can be seen in Figure 2, descriptive-subjective sentences, those sentences offering an opinion or evaluation of either the stimulus or the individual writing the essay, represent the largest proportion of sentences for both the Allen Griffen and the Cosby video. The pattern of descriptive-objective and problem-focused differed for the two videos, however, with problem-focused sentences more prevalent in the Allen Griffen essays and almost nonexistent in the Cosby essays.

\section{Immunological Data}

Correlations of precipitin ring measurements made by the two researchers for each of the four time periods ranged from .94 to .99, all significant at $p<.01$. Subsequent analyses involving these precipitin ring measurements as a measure of sIgA concentrations therefore utilize the mean of the two measurements made for each subject at each time period.

The correlation between subjects' sIgA scores across the four collection times and subjects' AIM scores was examined and found to be non-significant for both the Cosby 


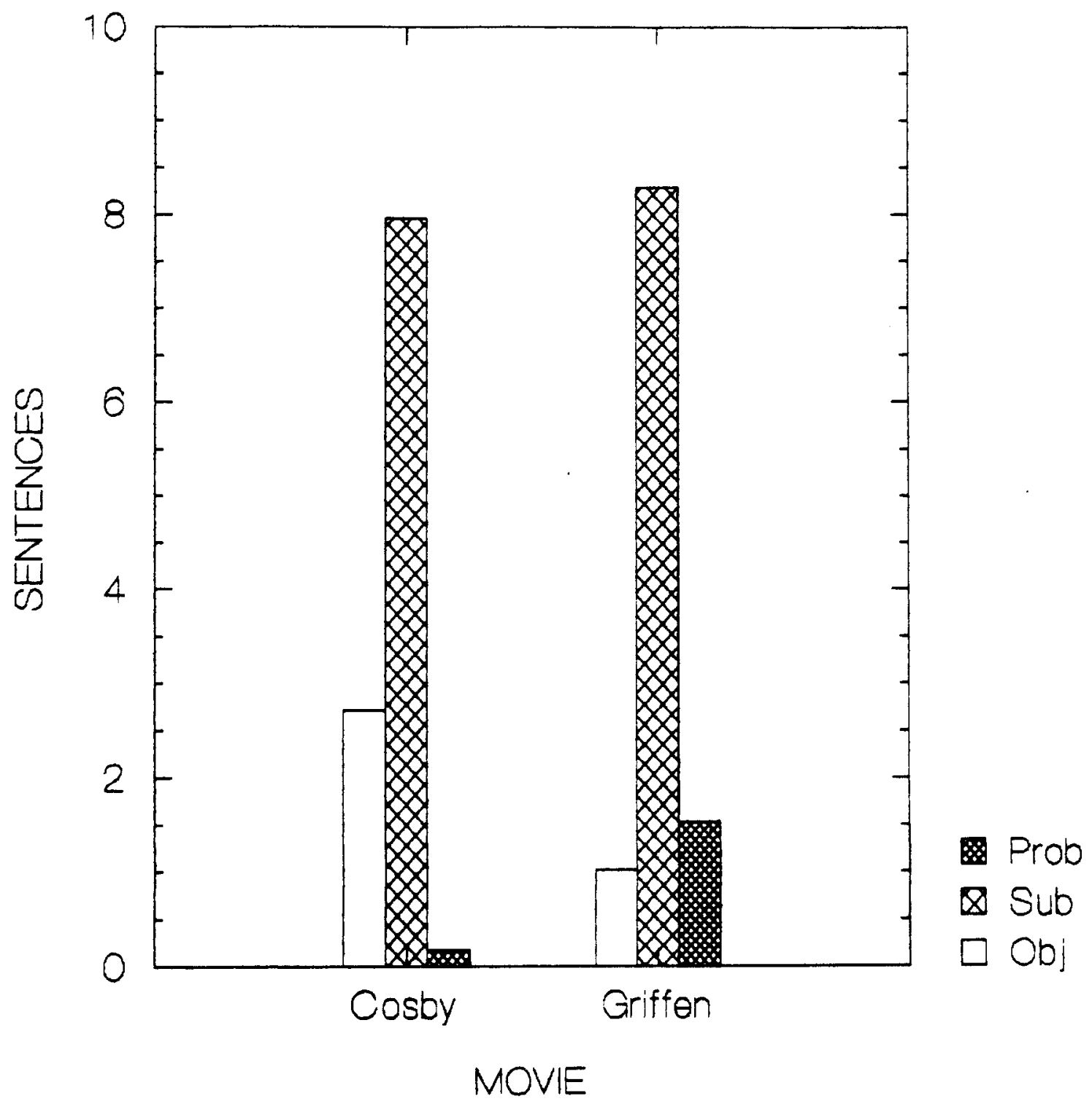

Figure 2 .

Mean number of types of sentences written by subjects writing essays about either the Cosby or Allen Griffen videos 
and the Allen Griffen videos (correlations ranged from .03 to -.29). These results indicate that affect intensity was not a reliable predictor of sIgA levels. These findings fail to support Hypothesis IV, and raise an important question: given that there was no affect intensity-sIgA link, is there an affect intensity-affect link as measured by the manipulation check? If so, the relationship between affect and SIgA is somewhat suspect. Such a link between affect and affect intensity appears to exist; AIM scores were significantly correlated with three post video manipulation check affect scores. Affect intensity was positively correlated with subjects' ratings of happiness after watching the Cosby video $(r=.62, p<.01)$, and with distress scores $(r=.45, p<.05)$ and anger scores $(r=.34$, $p<.05)$ after watching the Allen Griffen video.

Precipitin ring measurements for each subject were analyzed using a $2 \times 2 \times 2 \times 3$ repeated-measures analysis of covariance (ANCOVA), with type of video (positive/negative), essay topic (video/mundane topic), and gender as between groups variables, and saliva-collection times (immediately after writing essay/1 hour after writing essay/23 hours after writing essay) as a within-subjects variable. Pretest scores were used as a covariate to account for any posttest differences that were associated with pretest variance.

Initial, non-statistical inspection of the adjusted means for the four conditions, as seen in Table 2, indicates that sIgA concentrations appear to be similar for the time 
Table 2 .

Adjusted means of sIgA precipitin ring diameters (in millimeters) for both writing groups within the positive and negative affect groups.

\begin{tabular}{|c|c|c|c|c|}
\hline & $\begin{array}{l}\text { Writing } \\
\text { Tooic }\end{array}$ & $\begin{array}{c}\text { Immediately } \\
\text { After Writing }\end{array}$ & $\begin{array}{c}1 \text { Hour } \\
\text { After Writing }\end{array}$ & $\begin{array}{c}23 \text { Hours } \\
\text { After Writing }\end{array}$ \\
\hline & & & & Writing \\
\hline
\end{tabular}

Allen

Griffen

$\begin{array}{lllll}\text { Video } & \frac{n}{11} & 4.66 & 4.14 & 4.26 \\ \text { Shoes } & 14 & 4.66 & 4.26 & 4.08\end{array}$

Cosby

$\begin{array}{lllll}\text { Video } & 13 & 4.65 & 4.20 & 4.04 \\ \text { Shoes } & 12 & 4.49 & 4.17 & 4.02\end{array}$

Note. Higher scores represent greater concentrations of SIgA 
period immediately after writing for all the groups except the Cosby-shoes group, which had somewhat lower concentrations. Means for all conditions dropped across time, but the group that wrote about the Allen Griffen video seems to have rebounded slightly at the final testing period 24 hours after viewing the video.

The ANCOVA performed on these data revealed a main effect of writing, $F(1,42)=4.45, p=.041$, a main effect of time, $F(2,84)=31.74, p<.0001$, a time $x$ gender interaction, $F(2,84)=5.90, p=.004$, and a time $x$ movie $x$ gender interaction, $F(2,84)=4.60, p=.013$, as can be seen in Table 3 . The main effect for time and the time $\mathbf{x}$ gender interaction are thus interpretable only within the context of this three-way interaction. Inspection of adjusted means for the writing condition indicates that subjects who wrote about the video they watched, irrespective of which video it had been, showed higher sIgA levels than the subjects who had seen the videos and written about their shoes. The omegasquared for the writing effect, the proportion of variance in sIgA scores accounted for by the writing variable, was calculated to be .035 .

Follow-up inspection of the three-way interaction revealed that there was a gender difference across time for the positive affect (Cosby) video, $F=9.75(2,84), p=.0002$, but not for the negative affect (Allen Griffen) video, $F=.14$ $(2,84)$, n.s., such that males in the positive affect group started out (immediately after writing) with high sIgA 
Table 3 .

Source table for time by gender by movie by writing condition repeated measures ANCOVA on SIgA precipitin ring diameters $(\mathrm{N}=50)$

F p

\section{Between}

Movie

Write

Gender

Movie $x$ Write

Movie $x$ Gender

Write $x$ Gender

Movie $x$ Write $x$ Gender

Error

$\begin{array}{rlrrr}.0229 & 1 & .0229 & .05 & .8178 \\ 1.8947 & 1 & 1.8947 & 4.45 & .0411 \\ .3876 & 1 & .3876 & .91 & .3458 \\ .3572 & 1 & .3572 & .84 & .3653 \\ 1.0483 & 1 & 1.0483 & 2.46 & .1244 \\ 1.3411 & 1 & 1.3411 & 3.15 & .0835 \\ & & & & \\ .0522 & 1 & .0522 & .12 & .7282 \\ 17.4705 & 41 & .4261 & & \end{array}$

Within

Time

Time $x$ Movie

Time $x$ Write

$T$ ime $x$ Gender

$T$ ime $x$ Movie $x$ Write

$T$ ime $x$ Movie $x$ Gender

Time $x$ Write $x$ Gender

$T$ ime $x$ Movie $x$ Write $x$ Gender

Error
9.4697

.3916

.3033

1.7591

.0222

1.3710

.3 .676

.1941

12.5295

2
2
2
2
2

2

2

2

84
4.7349

.1958

.1517

.8796

.0111

.6855

.1838

.0970

.1491
1.23

.65

$\begin{array}{rr}31.74 & <.0001 \\ 1.30 & .2745 \\ 1.02 & .3662 \\ 5.90 & .0040 \\ .07 & .9283 \\ 4.60 & .0128 \\ 1.23 & .2969 \\ .65 & .5244\end{array}$


concentrations and then declined, while female concentrations started out lower but showed little change across time. Further investigation of the means for the positive affect video presented in Table 4 revealed a main effect of time for males, $F(2,84)=21.11, p<.0001$, but no main effect of time for females, $F(2,84)=1.71$, n.s. A Tukey HSD post hoc test was performed to examine the significant time effect for males. The test revealed significant differences between the immediately after writing and 1 hour after writing sIgA concentrations, and between immediately after writing and 23 hours after writing sIgA concentrations, but no significant difference existed between the concentrations at $1 \mathrm{hr}$ and 23 hours after writing.

One plausible explanation for the existence of the time main effect for men but not for women is that males found the Cosby video to be humorous while females did not (i.e., a gender $\mathbf{x}$ time interaction for the manipulations check measure). To test this possibility, separate gender $\mathbf{x}$ time repeated measures ANOVAs were performed on happiness, distress, sadness, and anger scores for the subjects who watched the Cosby video. The results indicated a main effect of time for each of the affects, as the earlier manipulation check inspection had discovered. There was also a marginal main effect of gender on anger scores, $F(1,23)=4.26, p=.0504$, suggesting that males were somewhat angrier than females both before and after watching the Cosby video. There were, however, no gender $x$ time 
Table 4 .

Adjusted means of sIgA precipitin ring diameters (in millimeters) for males and females who watched the positive affect (Cosby) video

\begin{tabular}{cccc} 
Gender & $\begin{array}{c}\text { Immediately } \\
\text { After Writing }\end{array}$ & $\begin{array}{c}1 \text { Hour } \\
\text { After Writing }\end{array}$ & $\begin{array}{c}23 \text { Hours } \\
\text { After Writing }\end{array}$ \\
$\begin{array}{c}\text { Male } \\
(n=8)\end{array}$ & 5.24 & 4.31 & 4.05 \\
$\begin{array}{c}\text { Female } \\
(n=17)\end{array}$ & 4.26 & 4.13 & 4.02 \\
\hline
\end{tabular}

Note. Higher scores represent greater concentrations of sIgA 
interactions for any of the affects, indicating that the Cosby video did not differentially affect males and females, at least with respect to these four affects. 


\section{Discussion}

The main effect for writing supports Hypotheses $I$ and II made at the outset. Within each video condition, the group that wrote about the video had higher SIgA concentrations than the control group. Though the amount of variance explained by writing was not great ( $3.5 \frac{\circ}{6}$ ), the fact that there was a reliable moderating effect on sIgA concentrations for writing about videos that could be viewed on television or rented on any given day is compelling.

Hypothesis III, in contrast, was not confirmed. This hypothesis predicted a time $x$ writing condition $x$ video interaction, with the Cosby-writing group having the highest sIgA concentration and the Allen Griffen-shoes group having the lowest sIgA concentration immediately after writing. Over time, levels were predicted to converge, until 23 hours after writing, when all groups were predicted to have the same sIgA concentrations. There was no interaction involving writing, however; overall, subjects who wrote about the video had higher sIgA concentrations than those who wrote about their shoes, across time and video.

As mentioned above, Hypothesis IV was not supported either. Affect intensity scores were not significantly correlated with SIgA concentrations at any of the four sIgA collection periods. One would expect that if affect exerts 
an influence on SIgA concentrations, affect intensity should mediate that relationship. Since affect intensity is correlated with affect and not sIgA concentrations, this calls into question the affect-sIgA link.

There are at least two possible explanations for this pattern of results. First, it is possible that some affect threshold had been reached by subjects watching the videos, a threshold beyond which the affect exerted no more influence on SIgA concentrations. I do not suggest that this is necessarily the highest affect threshold that exists, but simply that once this threshold is reached, the next threshold is beyond that which the video-generated affect could penetrate.

It is also possible that affect is not related to sIgA concentrations at all. Larsen and Diener (1987) have suggested that affect intensity may be one of several means of achieving an optimal level of autonomic arousal. People with low baseline levels of arousal would have high affect intensity to enable them to rise to optimal levels of arousal, while individuals high in baseline arousal would have low affect intensity to bring them down to optimal levels. This suggests that irrespective of the affect involved, affect intensity will work to generate the same, optimal levels of arousal in all subjects. This in turn suggests that the key to influencing sIgA concentrations is not affect, but autonomic arousal. 
While such a conclusion is plausible, it is not directly supportable given this experimental design. The first post-test SIgA collection was after subjects had written their essays, so it is not possible to determine whether it was the affect + writing manipulation or the autonomic arousal alone that precipitated sIgA changes. Previous research, however, casts doubt on the notion that simple autonomic arousal, irrespective of any positive or negative valence, is responsible for fluctuations in immunocompetence. Recall that Dillon et al. (1985-1986) and McClelland and Kirshnit (1988), found humor and feelings of love and caring to be associated with increased concentrations of SIgA. McClelland et al. (1982), KiecoltGlaser et al. (1984), Schleifer et al. (1983), and Jemmott and Magliore (1988) found that stress and negative affect were associated with immunodeficiency. Assuming that stress elevates autonomic nervous system levels, and humor and feelings of love do the same, the combination of these results argue against a simple arousal-sIgA link.

The three-way interaction involving sIgA levels as a function of gender, video, and time was unanticipated. Gender had not been expected to interact across time for either movie, and the fact that it did so for the positive affect movie, which apparently was no less affectively uplifting for females than males, is puzzling. It is possible that the humor in the Cosby video, humor presented by a male, was more germane to males than females. In other 
words, while both males and females found the video equally humorous, perhaps males found it more self-relevant and easier to internalize the humor, to process or interpret it in terms of their own experiences. This may have implications for how the affect impacts sIgA levels.

The lack of a main effect of video on SIgA concentrations is also surprising. Contrary to initial predictions, those who watched the humorous video, even those who wrote about the humorous video, did not exhibit higher SIgA concentrations than those who watched the negative affect video. The present results are especially baffling in light of evidence of the immunoenhancing effects of positive affect and the immunologically detrimental effects of negative affect, and given that the manipulation check data indicate that these videos generated the anticipated affect.

One possible explanation is the gender $x$ time interaction found in the Cosby video. As discussed above, female sIgA concentrations were lower than male concentrations after writing, and stayed low over time. This may explain, at least in part, why the Cosby video watchers as a whole did not exhibit higher sIgA concentrations than the Allen Griffen video watchers. This is especially problematic because the ratio of women to men was greater in the Cosby video condition (17:8) than in the Griffen video condition (14:11). Another possibility may have to do with 
the process underlying writing about the positive affect video. This will be discussed more fully below.

\section{Processes of Moderation}

The main effect for writing in this experiment is consistent with the results of experiments reviewed earlier that dealt with writing about positive and negative stimuli (e.g., McClelland \& Kirshnit, 1988; Pennebaker et al., 1988), and suggests that it is possible for a process or processes to moderate the impact of affect on immunocompetence. Since writing seems to attenuate the effect of stressful stimuli in one condition and to enhance or prolong the impact of positive stimuli in the other, there are at least two ways of viewing the act of writing. The first is that there is only one process underlying writing, that something about writing in and of itself leads to positive affect. Recall, however, that Pennebaker et al. (1988) found just the opposite with negative affect writing; subjects who wrote about negative personal experiences were less happy after writing than were control subjects. The second way in which writing about affective stimuli may be viewed is that writing entails two separate, distinct processes, one underlying writing about humorous or positive events, and another underlying writing about negative affect events. This conceptualization of what writing represents is logical and more consistent with the results of both the present study and past research in this area. 
McClelland and Kirshnit (1988) found that retaining or maintaining positive affect after watching a film about Mother Teresa was beneficial to immunocompetence. This is not the case with negative affect, however. Maintaining such negative affect has instead been associated with depressed immunocompetence (e.g., Pennebaker et al., 1988). The process underlying writing about negative stimuli, as Pennebaker and Beall (1986) and Pennebaker et al. (1988) suggest, most likely has something to do with expressing concerns that are somewhat stressful and that have not yet been discussed with anyone else, or even thought about by oneself at great length. In this context, writing may be thought of as a form of coping (Pennebaker, Colder, \& Sharp, 1990).

The coping process underlying writing about negative affect could perhaps be considered as cathartic in nature. This psychoanalytic concept suggests that merely venting one's emotions, "getting them off your chest," will have an immediate beneficial impact on immunology. Pennebaker et al.'s (1988) results, where subjects wrote about personally traumatic experiences, seem to belie this interpretation, however. Those subjects were significantly less happy than control subjects immediately after writing. A simple catharsis model would posit that subjects should experience greater relief and stronger positive affect immediately 
after writing. A cathartic/venting explanation of the writing process involved is thus found lacking.

Pennebaker $(1986 ; 1989)$ has suggested a theory of inhibition and confrontation to explain why writing (or talking, and in some cases thinking) about traumatic experiences is physiologically beneficial. According to this theory, individuals seek to understand their environment as a means of being able to predict and gain some control over it. When something happens, especially something self-relevant, an individual is compelled to seek some meaning from it, to assimilate information and experience, to construct some sort of order out of chaos. One can easily see how this is adaptive from an evolutionary point of view, both emotionally and cognitively.

From an information processing point of view, inhibition prevents people from fully processing the event they have experienced. Thoughts are purposely forced out of consciousness, restrained, held back so that they do not have to be considered. Inhibition prevents the assimilation of information and experiences that individuals need in order to gain a measure of perceived control over their environment. This inhibition is an active process, forcing back what naturally comes forward, and this active process requires physiological effort. This physiological work, over time and added to other stressors, can eventually lead to a taxing of the individual's system such that natural 
bodily activities, such as immune system functioning, can be impaired, resulting in somatic illness.

Support for the Theory

If, as Pennebaker suggests, inhibition requires physiological effort, then inhibition should result in detectable physiological variations. This, in fact, is precisely what research in this area has suggested. For example, Gray (1975), as cited in Fowles (1980), posits that specific regions of the brain are related to behavioral inhibition and that other regions of the brain are related to behavioral activation. Fowles (1980), building on Gray's model, provides evidence that behavioral inhibition is linked to electrodermal activity (EDA)--passive avoidance is associated with EDA changes, whereas active avoidance behavior is not. Pennebaker and Chew (1985) found that when subjects had to restrain naturally occurring behavior (inhibition), electrodermal activity increased. Lastly, Pennebaker, Hughes, and O'Heeron (1987) examined skin conductance levels (SCLs) during disclosure of traumatic events. SCLs decreased during disclosure for subjects labeled as high disclosers. Low disclosers, those who are unable or unwilling to disclose more traumatic experiences, in contrast, showed an increase in SCLs when they were supposed to be disclosing a traumatic experience. In other words, while the high disclosers disclosed, their SCLs dropped. While the low disclosers inhibited, on the other hand, their SCLs increased. Pennebaker's $(1986 ; 1989)$ 
theory of inhibition and confrontation has thus received empirical support, not only from research on immunocompetence, but from research dealing specifically with inhibition and autonomic functioning.

The Process Underlying Humor Writing

Unlike the process involved in negative affect writing, it seems unlikely that the immunologically beneficial effect of writing about the Bill Cosby video represents a process of coping with the positive affect. The process underlying writing about the humorous video most likely represents a prolonging of the positive impact on the immune system, rather than an immunoenhancement, or elevation of antibody/lymphocyte levels. In other words, I suggest that both groups who saw the Cosby video may have experienced an SIgA increase following the video, but only the group writing about the video maintained the elevated levels. It is impossible to test this given the design in this study, where no sIgA sample was collected immediately after viewing the video and immediately before writing (no saliva was collected here because the 5 minute saliva collection period might have interfered with the positive affect of the video, thus making the writing basically irrelevant, since there may have been no positive affect left to maintain). However, given the similar results obtained by McClelland and Kirshnit (1988) and by Dillon et al. (1985-1986), where watching a movie increased sIgA concentrations immediately 
after watching, it is reasonable to assume that something similar occurred here.

If. writing about the humorous video is fundamentally a prolonging of the positive affect derived from the video, then the moderating process between affect and immunocompetence in this case may simply be one of prolonging. If positive affect leads to enhanced immunocompetence, prolonged positive affect leads to prolonged enhanced immunocompetence. This process may be similar to what Bryant (1989) has termed savoring. Savoring is a process involving thoughts and behaviors aimed at intensifying or prolonging positive affect in response to past positive experiences, on-going positive experiences, and/or positive experiences that you anticipate happening in the future. Savoring is to positive stimuli as coping is to negative; instead of cognitively or behaviorally coming to terms with a traumatic or negative experience as one does in coping, one cognitively or behaviorally grasps positive experiences so as to better enjoy them. Writing about the pleasurable video stimulus may be a means of savoring what the stimulus has brought the individual.

Yet to call the process savoring without understanding what it is about the process that affects immunocompetence is little more than descriptive labeling. The same goes for calling writing about negative stimuli a "coping process." What is it about these savoring and coping processes that 
affects sIgA levels and presumably the somatic health of the individual?

Neuroimmunoendocrinology and the Moderating Processes Pennebaker's theory of inhibition rests on a relationship between inhibition and increased "work," which he posits is reflected by increased skin conductance levels while inhibiting. This "work" supposedly has an effect on immunocompetence. Skin conductance levels, however, are a reflection of the autonomic nervous system, while lymphocytes and sIgA are products of the immune system. What does this "work," which apparently affects the autonomic nervous system, really have to do with the immune system, and how is positive affect implicated in this matter?

One vital factor in this process is that the central nervous system (CNS) and the immune system are Iinked by the neuroendocrine system (Camara \& Danao, 1989). Neurons (part of the CNS) and lymphocytes (part of the immune system) share properties of certain cell targets of hormones. Specifically, catecholamines, a type of neurotransmitter which include hormones such as norepinephrine (NE) (Sarason \& Sarason, 1987), are released by the CNS and can be detected by lymphocytes. Lymphocytes, on the other hand, produce hormones which the CNS can detect.

This relationship is diagrammed in Figure 3. Catecholamines such as norepinephrine can have a two-part effect on lymphocytes (Madden \& Livnat, 1991). Initially, NE may enable lymphocyte activation, promoting antigen localization 


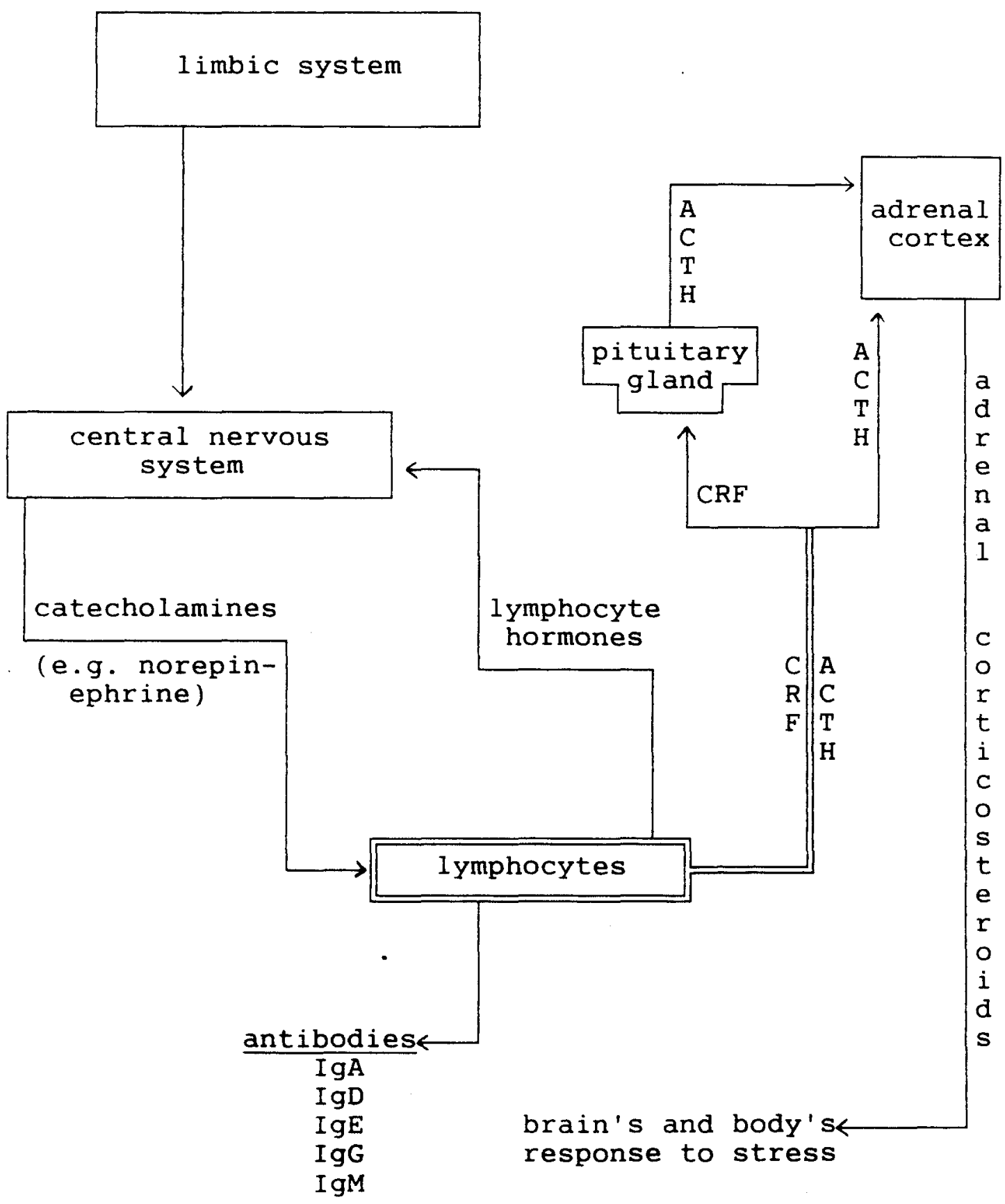

Figure 3 .

Conceptualization of the interaction among the immune, limbic, neuroendocrine, and central nervous systems 
and presentation, and B-cell activation (recall that it is $B$ lymphocytes that differentiate into plasmablasts and eventually into the plasma cells which are responsible for production of antibodies such as sIgA). Eventually, however, NE has an immunosuppressive effect, one of the consequences of which is diminished antibody production.

The CNS-immune system connection is not a one-way street, however. As mentioned above, lymphocytes produce hormones which affect the CNS. In addition, lymphocytes can secrete adrenocorticotropic hormone (ACTH) and corticotrophin-releasing factor (CRF). The CRF travels to the pituitary gland, stimulating the release of more ACTH. The ACTH proceeds to the adrenal cortex, causing the adrenal cortex to generate and release adrenal corticosteroids, which affect the body's response to stress (Sarason \& Sarason, 1987).

The CNS and the immune system thus engage in a two-way communication with each other, each affecting the other. Blalock (1984) cites research demonstrating that in addition to lymphocytes, the neuroendocrine system can exert influence on organs and tissues of the immune system, such as the spleen, the thymus gland, and bone marrow. The spleen stores some lymphocytes, and the thymus and bone marrow preprocess $\mathrm{T}$ lymphocytes and B lymphocytes, respectively (Guyton, 1991). It thus appears that the neuroendocrine system affects lymphocytes both directly as they 
circulate throughout the body and indirectly as or before they are preprocessed.

The final important piece to this puzzle concerns the limbic system. The limbic system mediates elements of affective and drive-motivated behavior (Camara \& Danao, 1989; Sarason \& Sarason, 1987). The limbic system also--and this is key to understanding the affect-immunocompetence relationship--"directly regulates" the autonomic and neurohumoral output of the CNS (Camara \& Danao, 1989, p.142). That is to say, the limbic system regulates the output of catecholamines (as well as other chemicals which impact the immune system), which we have seen above can have a direct influence on lymphocyte activity (including sIgA production), and hence immunocompetence.

Along these lines, MCClelland, Ross, and Patel (1985) have demonstrated the above mentioned relationship between the neuroendocrine system and the immune system. In a study on the effects of an academic examination, they analyzed SIgA and NE levels in saliva collected during a baseline time, immediately after a midterm examination, and one and three-quarters hour after the examination. Subjects had higher sIgA concentrations and NE levels immediately after and one and three quarters hours after the exam than they had at baseline. There was also a significant negative relationship between a) NE levels immediately and 1.75 hours after the exam and b) sIgA concentrations at the latter time period. In other words, the larger the increase in NE 
levels at the two time periods following the test. the lower the sIgA levels one and three-quarters hours after the test. This finding supports the research by Madden and Livnat (1991) cited above that NE levels eventually have an immunosuppressive effect.

Questions and Answers Regarding the Current Study

The studies above support Pennebaker's (1986; 1989) assertion that inhibition is related to physiological arousal. As mentioned above, Pennebaker asserts that over time, this arousal taxes both mind and body, eventually resulting in impaired immunocompetence. This assertion is borne out in his research where writing about personally traumatic situations leads to enhanced immunocompetence and to reduced health deficits (Pennebaker \& Beall, 1987; Pennebaker et al., 1988). Pennebaker et al. (1987) also offered as evidence for his theory the fact that SCLs increase with inhibition. Skin conductance levels are a reflection of the autonomic nervous system, and since the central nervous system has been shown to be linked to the immune system via the neuroendocrine system, this makes eminently good sense.

Additionally, the research concerning positive affect, savoring, and immunocompetence fits into place in light of the research on neuroimmunoendocrinology presented above. The limbic system, which mediates affective behavior, also regulates CNS release of catecholamines. A positive emotional state related to the limbic system could poten- 
tially influence the immune system via the central nervous system and the neuroendocrine system.

With respect to this study specifically, the main effect of writing makes sense as regards the preceding discussion of neuroimmunoendocrinology. Savoring--represented here by writing about the positive affect video-prolongs the effect the video has on the limbic system and the limbic system's subsequent influence on the neuroendocrine system. The coping process--represented in this research by writing about the negative affect article in Study I and the negative affect video in Study II--is instrumental in reducing inhibition of the negative stimuli. The corresponding lack of autonomic nervous system arousal impacts the neuroendocrine system. As has been discussed above, the neuroendocrine system, in both the savoring and the coping situations, then has either a positive or negative impact on lymphocytes and other aspects of the human immune system.

The lack of a main effect for the video condition is also interpretable after gaining an understanding of how the neuroendocrine system affects lymphocyte activity. Catecholamines, secreted by the central nervous system, do have a deleterious effect on lymphocyte activity, including antibody production. But before this adverse effect takes hold, there is an initial burst in lymphocyte capabilities. Recall the study conducted by McClelland et al. (1985) where sIgA concentrations initially increased after an exam, but 
NE levels were negatively correlated with sIgA concen-

trations later on. From an evolutionary perspective, this may represent the immune system's attempt, in conjunction with other systems of the body, to protect the body from some imminent threat. This may explain why unadjusted sIgA concentrations for subjects in both Study I and Study II (who watched the negative video) showed an increase in SIgA concentrations after writing, irrespective of the topic of their essay.

More questions than answers come to mind when trying to place the gender $\mathbf{x}$ time interaction for the Cosby video in the context of neuroimmunoendocrinology. The possibility was mentioned above that the humor may have been more germane to men than women, that men may have had an easier time interpreting the humor in terms of their own experiences. This presumably would entail a difference in levels of cognitive processing, and some amount of forebrain interaction with lower limbic system functioning. This is purely speculative and impossible to examine in this study. There is another plausible explanation for the observed differences. There may have been hormonal differences, stemming from female menstrual cycle activity, that intervened in the neuroendocrine system's relationship with the immune system (recall that catecholamines, the CNS secretions that directly affect lymphocyte activity, are hormones). Again, it is impossible to test this hypothesis in the current study. It is important to remember, however, 
that though there were gender differences with respect to the consequences of the positive affect video, these differences did not extend to the writing condition; writing had an effect for males and females in both video groups. Directions for Future Research

The studies presented here coupled with the review of contemporary research in this area support the necessity of adopting a biopsychosocial perspective to examine not only physical health but also psychological well-being. There is a definite link between affect and the functioning of the human immune system, a link mediated by the neuroendocrine system and moderated by psychological processes such as savoring and coping. Research in this area has only begun to investigate the incredible interactions and processes in which the human body engages on a routine, daily basis; the potential for further research is tremendous. There are, however, several specific avenues of study that follow from by this work.

The first area that merits attention is that of gender differences in immunological response to environmental stimuli, such as that found in the time $x$ gender interaction for the positive affect video. Do hormonal differences play a role here? Is there some other intervening variable that moderates the immunological responses of men and women, a variable on which men and women differ? Discovering the nature of such an intervening 
variable is important in any attempt to use environmental stimuli in a therapeutic manner.

Related to this is the issue of relevance of the environmental stimulus to the individual. Recall that Pennebaker et al. (1988) had their subjects write about personally traumatic experiences. In the present study, subjects wrote about experiences that were not their own, at least not directly. To the degree that the stimulus materials are personally relevant, is their a greater CNS change, and hence a greater immunological change? Cognitive psychology plays a role here in exploring what stimuli are more relevant to individuals and why, and how those stimuli are encoded, processed, stored.

A third avenue of future research concerns the role of inhibition in moderating the affect-immune system relationship. Pennebaker's $(1986 ; 1989)$ theory of inhibition and confrontation appears well-supported by his own research and by research on the CNS. A question remains, however. Is inhibition the sole culprit in reducing immunocompetence associated with negative affect, or does negative affect have an influence on immunocompetence beyond that of inhibition? In other words, if a person does not inhibit at a11, are there any negative ramifications of experiencing negative affect?

Lastly, what role, if any, does the immune system have in altering psychological states? Blalock (1984) argues that the immune system does not simply react to CNS activity 
but also instigates activity, and thus should be considered

a sensory organ. Recall that lymphocytes react to catecholamines, which are hormone neurotransmitters, but they also produce hormones which are detectable by the CNS (Camara \& Danao, 1989). Blalock posits that while cognitive stimuli are picked up by the CNS and result in immune system changes, information about noncognitive stimuli, such as viruses or bacteria, is sensed by the immune system and transported to the CNS, where autonomic changes may occur. It is, however, only one step from the CNS to the limbic system, and it is not outside the realm of consideration to postulate the influence of noncognitive stimuli on the immune system and subsequently on psychological functioning, at least at some basic level.

These are but a few of the many possible directions that research in the areas of psychological, social and biological processes (psychosocioneuroimmunoendocrinology?) may lead us. Whatever direction subsequent research takes, it is clear that it can only be in the general direction of an increasing understanding of the interrelation of these processes. To ignore the mind when researching somatic illness, to ignore the body when probing the mind and its psychological processes, to ignore social stimuli when exploring either of the other two domains, is to neglect the essence of what we as humans are and how we function. 


\section{$\underline{\text { References }}$}

Blalock, J.E. (1984). The immune system as a sensory organ. Journal of Immunology, 132, 1067-1070.

Bryant, F.B. (1989). A four-factor model of perceived control: avoiding, coping, obtaining, and savoring. Journal of Personality, 57(4), 773-797.

Camara, E.G. \& Danao, T.C. (1989). The brain and the immune system: A psychosomatic network. Psychosomatics, 30, 140-146.

De Toma, F.J. (1987). "Chapter 9: Enzyme Immunoassays." Experimental Immunology: A Guidebook. New York: Macmillan .

Dillon, K.M., Minchoff, B., \& Baker, K.H. (1985-1986). Positive emotional states and enhancement of the immune system. International Journal of Psychiatry in Medicine, 15, 13-17.

Fowles, D.C. (1980). The three arousal model:

Implications of Gray's two-factor learning theory for heart rate, electrodermal activity, and psychotherapy. Psychophysiology, 17, 87-104.

Gray, J.A. (1975). Elements of a two-process theory of learning. New York: Academic Press. Guyton, A. C. (1991). Textbook of Medical Physiology. Philadelphia: W. B. Saunders Company. 
Jemmott, J.B. \& Magloire, K. (1988). Academic stress,

social support, and secretory immunoglobulin A.

Journal of Personality and Social Psychology, 55, 803810.

Kiecolt-Glaser, J.K., Garner, W.K., Speicher, C., Penn, G.M., Holliday, J., \& Glaser, R. (1984). Psychosocial modifiers of immunocompetence in medical students. Psychosomatic Medicine, 46, 7-14.

Larsen, R.J. \& Diener, E. (1987). Affect intensity as an individual difference characteristic: A review. Journal of Research in Personality, 21, 1-39.

Lazarus, R.S. (1984). Puzzles in the study of daily hassles. Journal of Behavioral Medicine, $\underline{7}(4), 375-$ 389 .

Madden, K.S. \& Livnat, S. (1991). Catecholamine action and immunologic reactivity. In R. Ader, D.L. Felten, and N. Cohen (Eds.), Psychoneuroimmunology, 2nd ed. (pp. 283-310).

Martin, R.A. \& Dobbin, M.A. (1988). Sense of humor, hassles, and Immunoglobulin A: Evidence for a stressmoderating effect of humor. International Journal of Psychiatry in Medicine, 18, 93-105.

Martin, R.B., Guthrie, C.A., \& Pitts, C.G. (1991). Emotional crying, depressed mood, and immunity. Presented at American Psychological Association, San Francisco, CA. 
McClelland, D.C., Alexander, C., \& Marks, E. (1982). The need for power, stress, immune function, and illness among male prisoners. Journal of Abnormal Psychology, $91(1), 61-71$.

McClelland, D.C., Floor, E., Davidson, R.J., \& Saron, C. (1980). Stressed power motivation, sympathetic activation, immune function, and illness. Journal of Human Stress, $\underline{6}(2), 11-19$.

McClelland, D.C., \& Kirshnit, C. (1988). The effect of motivational arousal through films on salivary immunoglobulin A. Psychology and Health, 2, 31-52. McClelland, D.C., Ross, G., \& Patel, V. (1985). The effect of an academic examination on salivary norepinephrine and immunoglobulin levels. Journal of Human Stress, $\underline{2}, 52-59$.

Pennebaker, J.W. (1989). Confession, inhibition, and disease. In L. Berkowitz (Ed.), Advances in experimental social psychology (Vol. 4, pp. 211-242). San Diego: Academic Press.

Pennebaker, J.W. \& Beall, S.K. (1986). Confronting a traumatic event: Toward an understanding of inhibition and disease. Journal of Abnormal Psychology, 95, 274281 .

Pennebaker, J.W. \& Hoover, C.W. (1986). Inhibition and cognition: Toward an understanding of trauma and disease. In R. Davidson, G. Schwartz, and D. Shapiro 
(Eds.), Consciousness and self-regulation: Advances in research and theory, Vol. 4 (pp. 107-136).

Pennebaker, J.W., Hughes, C.F., \& O'Heeron, R.C. (1987).

The psychophysiology of confession: Linking inhibitory and psychosomatic processes. Journal of Personality and Social Psychology, 52, 781-793.

Pennebaker, J.W., Kiecolt-Glaser, J.K., \& Glaser, R. (1988).

Disclosure of traumas and immune function: Health implications for psychotherapy. Journal of Consulting and Clinical Psychology, 56, 239-245.

Pennebaker, J.W. \& O'Heeron, R.C. (1984). Confiding in others and illness rate among spouses of suicide and accidental-death victims. Journal of Abnormal

Psychology, 93, 473-476.

Rossen, R.D., Butler, W.T., Waldman, R.H., Alford, R.H., Hornick, R.B., Togo, Y., \& Kasel, J.A. (1970). The proteins in nasal secretion. Journal of the American Medical Association, 211, 1157-1161.

Sarason, I.G \& Sarason, B.R. (1987). Abnormal Psychology:

The Problem of Maladaptive Behavior. Englewood Cliffs: Prentice-Hall.

Schleifer, S.J., Keller, S.E., Camerino, M., Thornton, J.C., \& Stein, M. (1983). Suppression of lymphocyte stimulation following bereavement. Journal of the American Medical Association, 250(3), 374-377. Stone, A.A., Cox, D.S., Valdimarsdottir, H., Jandorf, L., \& Neale, J.M. (1987). Evidence that secretory IgA 
antibody is associated with daily mood. Journal of Personality and Social Psychology, 52(5), 988-993. Tijssen, P. (1985). Table 13.1. Practice and Theory of Enzyme Immunoassays. Amsterdam: Elsevier. Yodfat, Y. \& Silvian, H. (1977). A prospective study of acute respiratory tract infections among children in a kibbutz: The role of secretory IgA and serum immunoglobulins. The Journal of Infectious Diseases, $\underline{136}, 26-30$. 


\section{$\underline{\text { VITA }}$}

David Michael Njus was born in New Hampton, Iowa, in 1967. After growing up in rural lowa, he attended Luther College in Decorah, Iowa, where he graduated in 1990 with a bachelor of arts degree in psychology and English. He is currently attending Loyola University of Chicago, where he is pursuing a Ph.D. in applied social psychology. Mr. Njus first began his study of affect and immunological response at Luther College under the direction of Dr. Werner Nitschke. He continues this line of research at Loyola, working with Dr. Fred Bryant, and in continued collaboration with Nitschke.

In addition to this research and his other academic studies, Mr. Njus has been a research assistant at Loyola for two years, and will begin teaching undergraduate level psychology this summer. 
The thesis submitted by David M. Njus has been read and approved by the following committee:

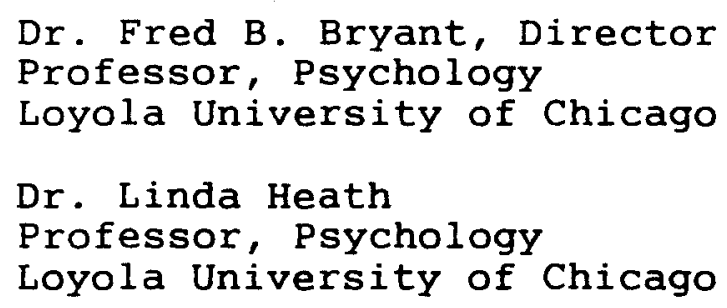

The final copies have been examined by the director of the thesis committee and the signature which appears below verifies the fact that any necessary changes have been incorporated and that the thesis in now given final approval by the committee with reference to content and form.

The thesis is, therefore, accepted in partial fulfillment of the requirements for the degree of Master of Arts.
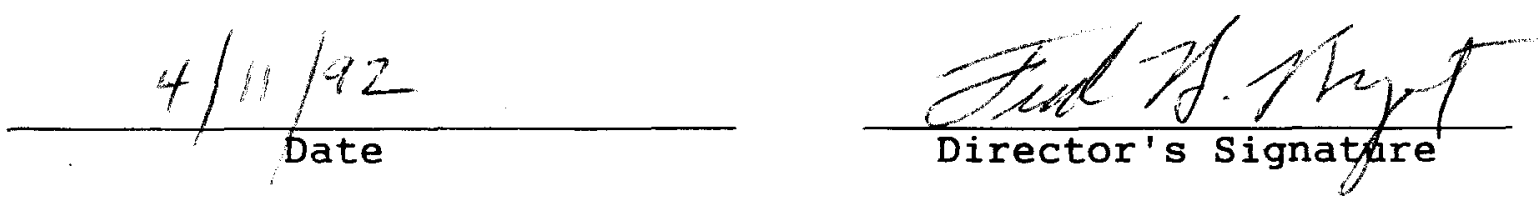